\title{
Dynamic simulation of land change in Western Chongqing based on SSO-CA model
}

\author{
Xiaoxiong Wen \\ School of Chongqing University of Posts and Telecommunications, Chongqing 400000, China; \\ wenxixo@outlook.com
}

Keywords: Computer application, SSO algorithm, Dynamic simulation of urban land.

\begin{abstract}
With China's rapid economic development, accelerate the process of city, with the city, how to use better planning of city land, the expansion of the city to better understand, improve the city geographic cellular automaton model based on accuracy, is the main research direction in this field. Aiming at the shortage of existing models, this paper constructs a SSO-CA model, and to the west of Chongqing area as an example of city land dynamic simulation, simulation results show that the total accuracy of SSO-CA model based on kappa was $81.4 \%$. The research showed that the simulation results than the genetic algorithm to construct the model based on SSO-CA model has better and more suitable for a large range of city land in dynamic simulation of uneven distribution of data, the data of the characteristic variables.
\end{abstract}

\section{Introduction}

With the series of problems brought by China's urbanization, the accelerated process of industrialization and the rapid growth of the population, the rational planning of urban land is required. How to achieve smart growth is an important part of regional sustainable development and smart city. The dynamic simulation of regional land use based on scenario assumption provides an important decision-making tool for urban and rural planners.

The cellular automaton model for order dynamical systems theory of turbulence, chaos, asymmetric, provides an effective tool ofmodel research overall behavior and the phenomenon of fractal system ${ }^{[1]}$, it can be used to simulate the transmission of information, calculation, structure, growth, reproduction, competition and evolution of many phenomena.

In recent years, CA has been widely used in dynamic simulation of urban ${ }^{[2]}$ because of its flexibility and discreteness. The flexibility of CA is reflected in its effective combination with machine learning methods, such as support vector machine and AI, to learn knowledge of past phenomena, so as to predict the future purpose of ${ }^{[3]}$. In recent years, many scholars at home and abroad have used traditional algorithms and intelligent algorithms to correct the CA model and excavate the rules of cell conversion.

The basic idea of bionic intelligent algorithm is to simulate the group behavior of natural organisms to construct a random optimization algorithm. It will search and optimization process of simulated evolution or foraging process, simulate the nature of individual points in the search space, the objective function for solving the problem of measuring individual ability to adapt to the environment into the process of survival of the fittest; individual or analogy for the foraging process with a good search and optimization of a feasible solution to replace the iteration the process is feasible. Therefore, the swarm intelligence algorithm is an iterative search algorithm with the feature of "generation + test". As a whole, the new intelligent computing technology is introduced into the field of urban land dynamic simulation. It will continue to replace the past traditional models and get wider application. It will provide new practice direction for the research of urban land dynamic simulation. With the help of the model, the spatio-temporal evolution of land use and cover change is revealed. It is of great significance to identify the ecological safety and promote the sustainable development of land. ${ }^{[4][5]}$.

The research on West Chongqing area as an example, try (1) by building simulation model of city dynamic combination of SSO and CA, and compared with the existing bionic intelligent algorithm 
model, test the effectiveness and accuracy of the simulation model; (2) to test the class imbalance produced influence on the simulation results (3; the city) scenario pattern simulation, revealed during the development of the city land use change development and internal rules, so as to city planning reasonable planning for the future sustainable development, land use distribution by city assistant.

\section{Research ideas and technical route}

This paper builds a SSO-CA model based on SSO algorithm ${ }^{[6]}$, which is used for dynamic simulation of urban land use. The technical route of city land dynamic simulation is mainly divided into three parts: (1) spatial data processing is completed, the main land use change detection and processing of spatial data by using the software of ARCGIS; (2) mining cellular rules, this part mainly uses the SSO algorithm and genetic algorithm to build mining model of cellular transformation rules; (3) using SSO-CA model and genetic algorithm model to simulate the land use change, and verify the accuracy of the simulation, mainly through Kappa coefficient verification, comparative analysis. The core part is the construction of the model and the training simulation part, and the regression coefficient of the corresponding spatial variables is obtained. The programming of the SSO-CA model and the genetic algorithm model is implemented mainly through the MATLAB.

In the constructed SSO-CA model, the fitness function of the group spider is designed for the classification accuracy of the sample data during the training process. The formula of fitness Fit is as follows:

$$
\text { Fit }=\frac{p_{1}+p_{2}}{p_{\text {all }}}
$$

$p_{1}$ and $p_{2}$ represent the correct number of records in the sample data set, respectively, for the construction land and the non construction land. $p_{\text {all }}$ represents the total number of records of the sample data set.

\section{Data sources}

Western Chongqing region, including 10 Yongchuan District, Jiangjin District, Hechuan District, Dazu area, Qijiang District, Rongchang District, Tongliang, Nanchuan District, Bishan District, Tongnan District, and the bridging Economic Development Zone, Wansheng economic development zone. The total area is 18828 square kilometers, 262 villages and towns (streets), the total (household registration) population is 10 million 403 thousand and 200, accounting for 31.36\% of Chongqing.

The west area is the core area of Chongqing City, the main economic, political and cultural function of Chongqing city is concentrated in this area. At the same time, this area is also an important area for the planning and development of the future government.

The main driving factors of the data selected elevation, slope, distance (the distance from the river from the main river and tributary distance), distance of market (distance from the city, district and Township Center), distance to road from the railway (distance from the two main road distance, road distance, distance from other Road), population density.

\section{Experimental results and comparative analysis}

In this paper, the SSO-CA model (scheme I) is used to compare the genetic algorithm model (scheme 2) to simulate the urban land use change. In this paper, the overall precision comparison method is used to evaluate the Kappa coefficient. In order to measure the accuracy of simulation results in terms of spatial morphology and overall structure, the Kappa coefficient is introduced to evaluate the simulation results, which can evaluate the accuracy of the simulation results from the aspects of spatial location, quantity and comprehensive information. In this paper, the SSO-CA model (scheme I) is used to compare the genetic algorithm model (scheme two) to simulate the urban land use change. The population size of the group spider algorithm in scheme 1 is set to 10 , the maximum iteration number is 100 times, the cross probability is 0.7 , the mutation probability is 0.1 . 


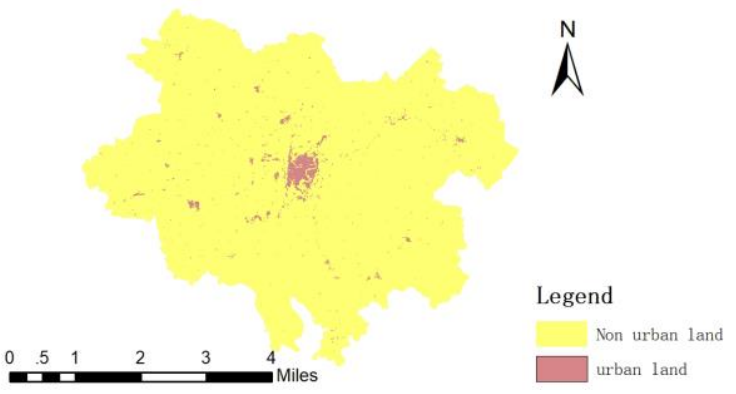

Fig. 1 SSO-CA Model Simulation effect diagram

The regression coefficient of the second scheme genetic algorithm regression model using maximum likelihood estimation method for parameter estimation, a program of genetic algorithm of population size is set to 10 , the maximum number of iterations for 100 times, the realization of adaptive crossover rate and mutation rate, the crossover rate is in the range of $[0.6,0.9]$, the mutation rate is in the range of $[0.03,0.15]$. Real number coding is used in the way of chromosome coding.

Table 1 Obfuscation matrix based on SSO-CA model

\begin{tabular}{|c|c|c|c|}
\hline Reality / simulation & Urban land & Non urban land & Total \\
\hline Urban land & 992364 & 2226 & 994590 \\
\hline Non urban land & 4848 & 16739 & 21587 \\
\hline Total & 997212 & 18965 & 1016177 \\
\hline
\end{tabular}

Table 2 Obfuscation matrix based on genetic algorithm

\begin{tabular}{|c|c|c|c|}
\hline Reality / simulation & Urban land & Non urban land & Total \\
\hline Urban land & 992192 & 2398 & 994590 \\
\hline Non urban land & 5746 & 15841 & 21587 \\
\hline Total & 997938 & 17879 & 1016577 \\
\hline
\end{tabular}

Table 3 Comparison of simulation accuracy of different methods

\begin{tabular}{|c|c|c|c|c|}
\hline Reality / simulation & \multicolumn{2}{|c|}{ SSO-CA } & \multicolumn{2}{c|}{ GA } \\
\hline & Urban land & Non urban land & Urban land & Non urban land \\
\hline accuracy & $86.5 \%$ & $99.5 \%$ & $85.3 \%$ & $99.7 \%$ \\
\hline Total precision & \multicolumn{2}{|c|}{$99.3 \%$} & \multicolumn{2}{c|}{$99.1 \%$} \\
\hline Total kappa & \multicolumn{2}{|c|}{$81.4 \%$} & \multicolumn{2}{c|}{$79.2 \%$} \\
\hline
\end{tabular}

Based on the obfuscation matrix generated by the SSO-CA model, as shown in Table 1, the final accuracy comparison of the two algorithm models is shown in Table 3. According to the calculation, we can see that the model based on the gregarious spider algorithm and cellular automata is more advantageous than the traditional genetic algorithm in the simulation accuracy and the total Kappa coefficient.

\section{Summary}

The research on Western Chongqing City as an example, the main land use types is simplified to the city and non city land, the introduction of social spider algorithm and cellular automaton model combining the trend of land use in Western Chongqing evolution simulation. In view of the traditional swarm intelligence algorithm in city land simulation of uneven distribution of data, classification problems, construct the social spider algorithm models are improved to a certain extent, through experiment, gregarious spider algorithm in the simulation of land use change, not only can get better optimization results, and the optimization of structural stability, a certain the degree of customer 
service of the genetic algorithm parameters are difficult to determine, the optimization result is not stable, to achieve a better simulation result, the total accuracy reached $92.3 \%$, the total Kappa reached $85.18 \%$.

\section{References}

[1]. Immanuel, W. Christopher; Deborrah, S. Paul Mary; Selvaraj, R. Samuel. Application of cellular automata approach for cloud simulation and rendering. CHAOS,2014,24(1).

[2]. Liu Xiaoping, Li Xia, Ye Jiaan, et al. Using ant colony intelligence to mine the transformation rules of geographic cellular automata [J]., 2007, 37 (6): 824-834.Liu Y, Phinn S R. Modelling urban development with cellular automata incorporating fuzzy-set approaches[J]. Computers Environment \& Urban Systems, 2003, 27(6):637-658.

[3]. Liu Y, Phinn S R. Modelling urban development with cellular automata incorporating fuzzy-set approaches[J]. Computers Environment \& Urban Systems, 2003, 27(6):637-658.

[4]. Teng Mingjun, Ceng Lixiong, Xiao Wenfa, et al. Research progress on remote sensing of ecological environment changes in the Three Gorges Reservoir area of the Yangtze River [J]. Applied Ecology journal, 2014, 25 (12): 3683-3693.

[5]. Cheng Hui, Wu Shengjun, Wang Xiaoxiao, et al. Research progress on ecological environmental effects of the Three Gorges Reservoir Area [J]. China ecologic agriculture journal, 2015 (2): 127-140.

[6]. Cuevas E,Cienfuegos M,Zaldívar D,et al. A swarm opti-mization algorithm inspired in the behavior of the social-spider [J] . Expert Systems with Applications, 2013,40( 16):6374 - 6384. 